Computing 9, 69-73 (1972)

(C) by Springer-Verlag 1972

\title{
Verfahren zur Berechnung des Spektralradius nichtnegativer irreduzibler Matrizen II
}

\author{
Von \\ L. EIsner, Erlangen \\ (Eingegangen am 11. November 1971)
}

Zusammenfassung - Summary

Verfahren zur Berechnung des Spektralradius nichtnegativer irreduzibler Matrizen II. In Ergänzung einer früheren gleichlautenden Arbeit werden weitere iterative Verfahren zur Berechnung des Spektralradius und des zugehörigen positiven Eigenvektors einer nichtnegativen irreduziblen Matrix angegeben und die Konvergenz bewiesen. Dabei streben die kleinsten Quotienten der Näherungsvektoren monoton gegen den Spektralradius. Es werden Einschließungsaussagen für den positiven Eigenvektor $y$ bewiesen und daraus die Konvergenz der Näherungen gegen $y$ hergeleitet.

Methods for computing the spectral radius of a non-negative irreducible matrix II. Supplementary to a former paper with the same title additional iterative methods for computing the spectralradius and the positive eigenvector of a nonnegative irreducible matrix are given and convergence is proved. Here the smallest quotients of the approximating vectors are monotously converging to the spectral radius. Inclusions for the positive eigenvector $y$ are given, from which the convergence of the approximations to $y$ is deduced.

\section{Einleitung}

In einer früheren Arbeit [2] wurde eine Klasse von Verfahren zur Berechnung des Spektralradius $\omega$ und des zugehörigen positiven Eigenvektors $y$ einer nichtnegativen irreduziblen $N \times N$-Matrix $A$ behandelt. Dabei wird, ausgehend von einem beliebigen positiven Vektor $x^{(0)}$, eine Folge von Näherungsvektoren $x^{(i)}$ fuir $y$ konstruiert. $x^{(i+1)}$ entsteht aus $x^{(i)}$ bis auf Normierung dadurch, daß diejenige Komponente von $x^{(i)}$, zu der der kleinste Quotient $(A x)_{k} / x_{k}$ gehört, mit einem Faktor $d_{i} \leq 1$ multipliziert wird. Unter einfachen Voraussetzungen an die Wahl der $d_{i}$ wurde in [2] Konvergenz der $x^{(i)}$ gegen $y$ gezeigt. Dabei konvergiert die Folge der größten Quotienten $R_{i}$ monoton gegen $\omega$. Hier wird nun das analoge Verfahren untersucht, bei dem jeweils die Komponente, zu der der größte Quotient gehört, vergrößert wird. Das ergibt eine Folge von Vektoren, deren kleinste Quotienten $r_{i}$ monoton steigen. Unter gewissen Voraussetzungen, die denen in [2] entsprechen, ist auch hier die Konvergenz beweisbar. Die Übertragung des Beweises von [2] ist aber nicht immer möglich.

Im zweiten Abschnitt (Satz 1) wird gezeigt, daß unter den erwähnten Voraussetzungen die $r_{i}$ gegen $\omega$ streben. Im dritten Abschnitt werden Einschließungsaussagen für den Eigenvektor $y$ hergeleitet, die einige in der Literatur vorliegende Abschätzungen verbessern. Der angekündigte Konvergenzsatz folgt ebenfalls daraus.

Die Bezeichnungen entsprechen denen in [2]. 


\section{Eine Klasse von Verfahren}

Es sei $A$ eine nichtnegative irreduzible $N \times N$-Matrix, $\omega=\rho(A)$ der Spektralradius von $A$ und $y$ der durch $\|y\|=1\left(\|\ldots\|\right.$ irgendeine Norm in $\left.R^{N}\right)$ eindeutig festgelegte positive Eigenvektor: $A y=\omega y . \quad x \leq z\left(x, z \in R^{N}\right)$ bedeute $x_{i} \leq z_{i}$ für $i=1, \ldots, N$. Zu gegebenem positiven Vektor $x=\left(x_{1}, \ldots, x_{N}\right)>0(\mathrm{~d} . \mathrm{h}$. $\left.x_{i}>0, i=1, \ldots, N\right)$ sei

$$
R_{i}(x)=(A x)_{i} / x_{i}=\sum_{k=1}^{N} a_{i k} x_{k} / x_{i}
$$

der $i$-te Quotient. $(i=1, \ldots, N$.) Bekanntlich gilt nach dem Quotientensatz [1] für jedes $x>0$

$$
\min _{k} R_{k}(x) \leq \omega \leq \max _{k} R_{k}(x)
$$

Ausgehend von $x^{0}>0$ konstruieren wir eine Folge positiver Vektoren $x^{i}=\left(x_{1}^{i}, \ldots, x_{N}^{i}\right)$ mit zugehörigen Quotienten $R_{k}^{i}$ folgendermaßen:

Sei $v=v(n), \mu=\mu(n)$ so gewählt, daß

$$
R_{v}^{n} \leq R_{i}^{n} \leq R_{\mu}^{n}
$$

für $i=1, \ldots, N$ gilt. Mit noch zu bestimmenden Größen $d_{n}$ sei

$$
\begin{aligned}
& \tilde{x}_{k}^{n+1}=\left\{\begin{array}{rr}
x_{k}^{n} & k \neq \mu(n) \\
d_{n} x_{k}^{n} & k=\mu(n)
\end{array}\right. \\
& x^{n+1}=\tilde{x}^{n+1} /\left\|\tilde{x}^{n+1}\right\| .
\end{aligned}
$$

Es sei $r_{n}=R_{v}^{n}=\min R_{i}^{n}$. Dann gilt

Satz 1. Sei $d_{n}$ so gewählt, daß $(a),(b),(c)$ erfüllt sind:

(a) $R_{v}^{n}<R_{\mu}^{n} \Rightarrow d_{n}>1$.

(b) $\exists\{n(i)\}, \lim _{i} d_{n(i)}=1 \Rightarrow \lim r_{n}=\omega$.

(c) $\exists \alpha: 0<\alpha<1 \mathrm{mit}$

$$
R_{\mu(n)}^{n+1} \geq \alpha R_{\mu(n)}^{n}+(1-\alpha) R_{v(n)}^{n}
$$

Dann ist $\lim r_{n}=\omega$.

Beweis. Da die Quotienten homogene Funktionen des Vektors $x$ sind, kann beim Beweis die Normalisierung (3) unbeachtet bleiben. Wegen (a) ist bei dieser Verabredung $x_{k}^{n}$ für jedes $k$ in $n$ monoton nicht fallend. Allgemein gilt $(\mu=\mu(n))$ :

$$
\begin{aligned}
& R_{i}^{n+1}=R_{i}^{n}+\left(d_{n}-1\right) a_{i \mu} x_{\mu}^{n} / x_{i}^{n} \quad i \neq \mu \\
& R_{\mu}^{n+1}=a_{\mu \mu}+\left(R_{\mu}^{n}-a_{\mu \mu}\right) / d_{n}
\end{aligned}
$$

Zusammen mit (a) und (c) folgt

$$
r_{1} \leq \ldots \leq r_{n} \leq r_{n+1} \ldots \leq \omega .
$$

Sei $I=\{p \in\{1, \ldots, N\}, p \neq \mu(n)$ für alle hinreichend großen $n\}$. Wir machen nun eine Fallunterscheidung. 
Fall 1. $I \neq \Phi$. Offenbar ist auch $J=\{1, \ldots, N\} \div I \neq \Phi$. Wegen der Irreduzibilität von $A$ gibt es $k \in I, l \in J, a_{k l}>0$. Für hinreichend großes $n$ ist wegen $\mu(n) \neq k$ stets

$$
R_{k}^{n+1}=R_{k}^{n}+\left(d_{n}-1\right) a_{k \mu(n)} x_{\mu(n)}^{n} / x_{k}^{n}
$$

und $x_{k}^{n}$ konstant. Insbesondere ist $a_{k l} x_{l}^{n} / x_{k}^{n}$ nach unten beschränkt.

Fall 1 a. Es gibt eine Teilfolge $n(i)$ mit $d_{n(i)} \rightarrow 1$. Nach (b) folgt die Behauptung.

Fall 1 b. Es gibt $\varepsilon>0$ mit $d_{n} \geq 1+\varepsilon$ für alle $n$. Da immer wieder $\mu(n)=l$ ist, folgt nach (5) $\lim R_{k}^{n}=\infty$.

Für $s \in J$ ist $\lim x_{s}^{n}=\infty$. Daher gibt es $s \in J$, so $\mathrm{da} \beta x_{s}^{n}=\max _{i} x_{i}^{n}$ für abzählbar viele $n$ gilt.

Sei $M=\sum_{j} a_{s j}$ und $N_{0}$ so groß, daß $R_{k}^{n}>M \alpha^{-1}$ für alle $n \geq N_{0}$ ist. Ist $x_{s}^{n}$ maximal, so ist $R_{s}^{n} \leq \sum_{j} a_{s j}=M$, für $n \geq N_{0}$ ist dann also $\mu(n) \neq s$. Anders gewendet: Ist $\mu(n)=s$, so ist $x_{s}^{n}$ nicht maximal. Es gibt daher $n \geq N_{0}$ mit $x_{s}^{n}$ nicht maximal, aber $x_{s}^{n+1}$ maximal. Dann ist wegen der Monotonie $\operatorname{der} x_{j}^{n}$ sicher $x_{s}^{n+1}>x_{s}^{n}$, also $\mu(n)=s$ und wegen (c) $R_{s}^{n+1} \geq \alpha R_{s}^{n}$. Daher

$$
M \alpha^{-1}<R_{k}^{n} \leq R_{s}^{n} \leq \alpha^{-1} R_{s}^{n+1} \leq M \alpha^{-1} .
$$

Fall 1 b) tritt also nicht auf.

Fall 2. $I=\Phi$. Dieser Fall wird völlig analog zu [2] behandelt und nur der Vollständigkeit halber aufgeführt. Es gibt ein $p$, so daß für abzählbar viele $n$ gilt: $v(n)=p$. Dann gibt es Zahlenfolgen $m(i), n(i)$ mit

$$
\begin{aligned}
& m(i)<n(i)<m(i+1), \mu(m(i))=v(n(i))=p \quad i=1,2, \ldots, \\
& m(i)<n<n(i) \Rightarrow \mu(n) \neq p .
\end{aligned}
$$

Dann gilt $R_{p}^{m(i)+1} \leq R_{p}^{n(i)}=r_{n(i)}$, also $0 \leq \omega-r_{n(i)} \leq \omega-R_{p}^{m(i)+1} \leq \alpha\left(\omega-R_{p}^{m(i)}\right)+(1-\alpha)\left(\omega-R_{v(m(i)}^{m(i)}\right) \leq(1-\alpha)\left(\omega-r_{m(i)}\right)$

Zusammen mit der Monotonie der $r_{i}$ folgt daraus $\lim r_{n}=\omega$. Q.E.D.

\section{Einschließung des Eigenvektors}

Grundlage der folgenden Einschließungen ist das

Lemma. Sei $A \geq 0, x>0, z>0, A x \leq \alpha x, A z \geq \beta z, x_{i} / z_{i}=\min x_{j} / z_{j}$. Ist $k$ ein Index mit $a_{i k}>0$, so gilt

$$
\begin{aligned}
& \frac{x_{i}}{z_{i}} \leq \frac{x_{k}}{z_{k}} \leq\left(1+\frac{\alpha-\beta}{a_{i k}} \frac{z_{i}}{z_{k}}\right) \frac{x_{i}}{z_{i}} \\
& \left(1-\frac{\alpha-\beta}{a_{i k}} \frac{x_{i}}{x_{k}}\right) \frac{z_{i}}{x_{i}} \leq \frac{z_{k}}{x_{k}} \leq \frac{z_{i}}{x_{i}} .
\end{aligned}
$$

Beweis. Aus

$$
\alpha-\beta \geq(A x)_{i} / x_{i}-(A z)_{i} / z_{i}=\sum_{j} a_{i j} \frac{z_{j}}{z_{i}}\left(\frac{x_{j} z_{i}}{x_{i} z_{j}}-1\right)
$$


und $\frac{z_{i} x_{j}}{x_{i} z_{j}} \geq 1(j=1, \ldots, N)$ folgt

$$
a_{i k} \frac{z_{k}}{z_{i}}\left(\frac{x_{k} z_{i}}{x_{i} z_{k}}-1\right) \leq \alpha-\beta
$$

und daraus (6). Eine triviale Umformung ergibt (7).

Folgerung 1. Ist $A>0, x=y$ der Eigenvektor zum Spektralradius $\omega$, $z=(1, \ldots, 1)$, so sind die Voraussetzungen des Lemmas mit $\alpha=\omega, \beta=r=\min _{i} \sum_{k} a_{i k}$ erfüllt. (6) ergibt nun

$$
\max _{j, k} \frac{y_{j}}{y_{k}} \leq 1+\frac{\omega-r}{m}
$$

wobei $m=\min \left\{a_{j k} \mid j \neq k\right\}$ ist.

Wegen $\omega \leq R=\max _{i} \sum_{k} a_{i k}$ folgt daraus sofort eine Abschätzung von OSTROWSKI [5]

$$
\operatorname{Max}_{j, k} \frac{y_{j}}{y_{k}} \leq 1+\frac{R-r}{m}
$$

Folgerung 2. Sei $A \geq 0$ irreduzibel, $x>0, z>0, A x \leq \alpha x, A z \geq \beta z, x_{i} / z_{i}=\min !$, $m=\min \left\{a_{i k} \mid a_{i k}>0, i \neq k\right\}$. Zu gegebenem $k$ gibt es $s \leq N-1$ mit $a_{i k}^{(s)}=\left(A^{s}\right)_{i k}>0$. Wendet man das Lemma auf $A^{s}$ an, so ergibt (6) bzw. (7):

$$
\begin{aligned}
& \frac{x_{i}}{z_{i}} \leq \frac{x_{k}}{z_{k}} \leq\left(1+\frac{\alpha^{s}-\beta^{s}}{a_{i k}^{(s)}} \frac{z_{i}}{z_{k}}\right) \frac{x_{i}}{z_{i}} \\
& \left(1-\frac{\alpha^{s}-\beta^{s}}{a_{i k}^{(s)}} \frac{x_{i}}{x_{k}}\right) \frac{z_{i}}{x_{i}} \leq \frac{z_{k}}{x_{k}} \leq \frac{z_{i}}{x_{i}}
\end{aligned}
$$

Mit $z=(1, \ldots, 1)$ ergibt $(8)(r$ wie in Folgerung 1)

und damit

$$
\frac{x_{k}}{x_{i}} \leq 1+\frac{\alpha^{s}-r^{s}}{m^{s}}
$$

$$
\max _{j, k} \frac{x_{k}}{x_{j}} \leq 1+\frac{\alpha^{N-1}-r^{N-1}}{m^{N-1}}
$$

Das verbessert eine Abschätzung von SCHNEIDER [6]

$$
\max _{j, k} \frac{x_{k}}{x_{j}} \leq\left(\frac{\alpha}{m}\right)^{N-1}
$$

Als dritte Folgerung beweisen wir

Satz 2. Unter den Voraussetzungen von Satz I gilt

$$
\begin{aligned}
& \lim _{n} x^{n}=y \\
& \lim _{n} R_{k}^{n}=\omega \quad k=1, \ldots, N
\end{aligned}
$$


Beweis. Wir verwenden (9) für $x=y, \alpha=\omega, z=x^{n}, \beta=r_{n}$ und erhalten wegen $\lim r_{n}=\omega$ (nach Satz 1) für beliebiges $j, k$

$$
\lim _{n} \frac{x_{k}^{n}}{y_{k}} \cdot \frac{y_{j}}{x_{j}^{n}}=1 .
$$

Jeder Häufungspunkt der $x^{n}$ ist wegen (12) und der Normierung $\left\|x^{n}\right\|=1$ gleich $y$. Das zeigt (10) und (11). Q.E.D.

\section{Ein Beispiel}

Die Klasse der Verfahren, die die Voraussetzungen von Satz 1 und 2 erfüllen, ist nicht leer. Den in [2] angegebenen Verfahren lassen sich jeweils analoge Verfahren der hier beschriebenen Art zur Seite stellen. Dem Verfahren 1 entspricht hier bei gegebenem $0<\alpha<1$ die Vorschrift

$$
d_{n}=\left\{\begin{array}{cl}
\frac{R_{\mu}^{n}-a_{\mu \mu}}{\alpha R_{\mu}^{n}+(1-\alpha) R_{v}^{n}-a_{\mu \mu}} & \text { falls der Nenner positiv ist } \\
d & \text { sonst }
\end{array}\right.
$$

wobei $d>1$ fest gewählt ist. Man sieht leicht, daß (a)-(c) erfüllt sind. $d_{n}=d$ kann nur zu Beginn auftreten. Ebenso lassen sich die Verfahren 2 und 3 von [2] übertragen, wenn man wie oben gewisse Abänderungen vornimmt, falls $d_{n}$ unendlich oder negativ wird. Das obige Verfahren liefert für die in [2] angegebene Matrix für $\alpha=0,5$ den Spektralradius bereits nach 13 Schritten mit einer Genauigkeit von $10^{-4}$, wogegen das entsprechende Verfahren in [2] dazu 16 Schritte benötigt.

\section{Literatur}

[1] Collatz, L.: EinschlieBungssatz für die charakteristischen Zahlen von Matrizen. Math. Z. 48, $221-226(1942)$.

[2] Elsner, L.: Verfahren zur Berechnung des Spektralradius nichtnegativer irreduzibler Matrizen. Computing 8, 32-39 (1971).

[3] Hall, C. A., und T. A. Porsching: Computing the maximal eigenvalue and eigenvector of a positive matrix. SIAM J. Num. Anal. 5, 269-274 (1968).

[4] HALI, C. A., und T. A. Porsching: Computing the maximal eigenvalue and eigenvector of a nonnegative irreducible matrix. SIAM J. Num. Anal. 5, 470-474 (1968).

[5] Ostrowski, A.: Bounds for the greatest latent root of a positive matrix. J. London Math. Soc. 27, 253-256 (1952).

[6] SCHNeIDER, H.: Note on the fundamental theorem on irreducible non-negative matrices. Proc. Edinburgh Math. Soc. 11, 127-130 (1958/59).

\section{Ludwig Elsner}

Institut für Angewandte Mathematik I

der Universität Erlangen

Erwin-Rommel-Straße 60

D-852 Erlangen, Deutschland 